\title{
Five genes may predict metastasis in non-small cell lung cancer using bioinformatics analysis
}

\author{
RUIYING SUN, XIA MENG, WEI WANG, BOXUAN LIU, XIN LV, JINGYAN YUAN, \\ LIZHONG ZENG, YANG CHEN, BO YUAN and SHUANYING YANG \\ Department of Respiratory and Critical Care Medicine, \\ The Second Affiliated Hospital of Xi'an Jiaotong University, Xi'an, Shaanxi 710004, P.R. China
}

Received July 6, 2018; Accepted May 14, 2019

DOI: $10.3892 / \mathrm{ol} .2019 .10498$

\begin{abstract}
Lung cancer is one of the most common types of malignancy worldwide. The prognosis of lung cancer is poor, due to the onset of metastases. The aim of the present study was to examine lung cancer metastasis-associated genes. To identify novel metastasis-associated targets, our previous study detected the differentially expressed mRNAs and long non-coding RNAs between the large-cell lung cancer high-metastatic 95D cell line and the low-metastatic 95C cell line by microarray assay. In the present study, these differentially expressed genes (DEGs) were analyzed via bioinformatics methods, including Gene Ontology functional analysis and Kyoto Encyclopedia of Genes and Genomes pathway enrichment analysis. A protein-protein interaction network was subsequently constructed using the Search Tool for the Retrieval of Interacting Genes/Proteins online database and Cytoscape software, and 17 hub genes were screened out on the basis of connectivity degree. These hub genes were further validated in lung adenocarcinoma (LUAD) and lung squamous cell carcinoma (LUSC) using the online Gene Expression Profiling Interactive Analysis database. A total of seven hub genes were identified to be significantly differentially expressed in LUAD and LUSC. The prognostic information was detected using Kaplan-Meier plotter. As a result, five genes were revealed to be closely associated with the overall survival time of patients with lung cancer, including phosphoinositide-3-kinase regulatory subunit 1 , FYN, thrombospondin 1 , nonerythrocytic $\alpha$-spectrin 1 and secreted phosphoprotein 1 . In addition, lung cancer and adjacent lung tissue samples were used to validate these hub genes by reverse transcription-quantitative polymerase chain reaction. In conclusion, the results of the present study may provide
\end{abstract}

Correspondence to: Dr Shuanying Yang, Department of Respiratory and Critical Care Medicine, The Second Affiliated Hospital of Xi'an Jiaotong University, 157 Xiwu Road, Xi'an, Shaanxi 710004, P.R. China

E-mail: yangshuanying66@163.com

Key words: lung cancer, metastasis, bioinformatics analysis novel metastasis-associated therapeutic strategies or potential biomarkers in non-small cell lung cancer.

\section{Introduction}

Lung cancer is one of the most common causes of malignancy-associated mortality globally (1). Non-small cell lung cancer (NSCLC) accounts for $>80 \%$ of primary lung cancer cases (2). Despite improvements in traditional and novel treatments, including surgical resection, chemotherapy, radiotherapy and targeted therapy, the prognosis for patients with lung cancer remains poor, with a 5-year overall survival (OS) rate of $<20 \%$, due to a high frequency of metastasis (3). Therefore, the prevention and treatment of tumor metastasis are particularly important.

Gene expression microarray technologies have been widely used to identify the functional variation of the transcriptome in different cell types and tissues (4). A key advantage of microarray technology is that it can simultaneously and comprehensively detect the expression of tens of thousands of genes. Through gene chips, genes that may be associated with a disease can be identified in a short period of time, which may reveal biomarkers for early diagnosis or targeted therapy (5).

To identify novel metastasis-associated targets, our previous study detected differentially expressed mRNAs and long non-coding RNAs between the large-cell lung cancer high-metastatic 95D cell line and the low-metastatic 95C cell line using a microarray assay (6). A total of 252 mRNAs were screened out according to the cut-off criteria. Among them, 120 mRNAs were revealed to be upregulated, while 132 mRNAs were downregulated in 95D cells compared with 95C cells.

In the present study, these differential expressed genes (DEGs) were analyzed by a series of bioinformatics methods, including Gene Ontology (GO) functional analysis, Kyoto Encyclopedia of Genes and Genomes (KEGG) pathway enrichment analysis and protein-protein interaction (PPI) network construction. The hub genes were subsequently analyzed by Gene Expression Profiling Interactive Analysis (GEPIA) and Kaplan-Meier plotter (KM plotter) online databases. Furthermore, lung cancer tissues from patients who underwent surgery were used to further verify the results. Overall, the aim of the present study was to identify hub genes that may be involved in the process of lung cancer metastasis. 


\section{Materials and methods}

Data preprocessing. The raw microarray data from our previous study (6) was utilized in the present study. The fold-changes (FCs) in the expression of individual mRNAs between the 95D and 95C cell lines were calculated. Statistically significant differentially expressed mRNAs were defined as $\mathrm{P}<0.05$ and $\log 2|\mathrm{FC}|>2.0$. The genes that corresponded to these mRNAs were identified according to the National Center for Biotechnology Information (NCBI) database (https://www.ncbi.nlm.nih.gov/).

GO functional enrichment and KEGG pathway analysis. GO functional enrichment analysis was performed using the GO online database (http://www.geneontology.org) and the Database for Annotation, Visualization and Integrated Discovery (DAVID 6.7) online database (https://david.ncifcrf. gov/) (7). Pathway analysis was performed using the KEGG database (http://www.genome.jp/kegg). The P-value denotes the significance of the pathway associated with the conditions. The lower the $\mathrm{P}$-value, the more significant the pathway. $\mathrm{P}<0.05$ was considered to indicate a statistically significant result.

Construction of a PPI network and hub gene identification. In order to detect the potential associations among those DEGs, the STRING version 10.5 database (https://www.string-db. org/) and Cytoscape 3.6.1 software (http://www.cytoscape. org/) were used to construct a PPI network. The cut-off criteria in the STRING database was set as: Confidence score $\geq 0.4$ and maximum number of interactors $=0$. In addition, Cytoscape plug-ins, including Molecular Complex Detection (MCODE) and cytoHubba, were utilized to screen modules of the PPI network and hub genes, respectively. The criteria in MCODE was set as: Degree cut-off $=2$, node score cut-off $=0.2, \mathrm{k}$-core $=2$ and max. depth=100. The hub genes were defined as the top 17 nodes ranked by degree in cytoHubba.

Expression levels of the hub genes in the cancer genome atlas (TCGA) database. GEPIA (http://gepia.cancer-pku. $\mathrm{cn} /$ index.html) contains the RNA sequencing expression data of 9,736 tumors and 8,587 normal cases from TCGA and the Genotype-Tissue Expression (GTEx) projects (8), and was used in the current study to compare the expression levels of the hub genes between lung cancer tissues and normal tissues. Boxplots were subsequently generated to visualize the associations.

Survival analysis of hub genes. In the present study, KM plotter online database (http://kmplot.com/analysis) was used to evaluate the prognostic value of the hub genes. KM plotter can be used to assess the effect of 54,675 genes on survival using 10,461 cancer samples, including 5,143 patients with breast cancer, 1,816 with ovarian cancer, 2,437 with lung cancer and 1,065 with gastric cancer, with a mean follow-up of 69, 40, 49 and 33 months, respectively (9). The relapse-free time and OS time information were based on GEO (Affymetrix microarrays), European Genome-phenome Archive (https://www.ebi.ac.uk/ega/home) and TCGA databases (https://portal.gdc.cancer.gov/). In GEO, the optimal Affymetrix IDs were 212239_at (PIK3R1),212486_s_at (FYN), 201110_s_at (THBS1), 215235_at (SPTAN1) and 48580_at
Table I. Primer sequences.

\begin{tabular}{ll}
\hline Gene & \multicolumn{1}{c}{ Sequences $\left(5^{\prime} \rightarrow 3^{\prime}\right)$} \\
\hline GAPDH & F: GTCTCCTCTGACTTCAACAGCG \\
& R:ACCACCCTGTTGCTGTAGCCAA \\
PIK3R1 & F: ACCACTACCGGAATGAATCTCT \\
& R:GGGATGTGCGGGTATATTCTTC \\
FYN & F: GAAGCACGGACAGAAGATGACCTG \\
& R:CACCAATCTCCTTCCGAGCTGTTC \\
SPTAN1 & F: TGCTTGCTGCTGGTCACTATGC \\
& R:GAACGCCTCCTGCTTGCTCATC \\
THBS1 & F: GGCACCAACCGCATTCCAGAG \\
& R:GCACAGCATCCACCAGGTCTTG \\
SPP1 & F: AGCGAGGAGTTGAATGGTGCATAC \\
& R:AATCTGGACTGCTTGTGGCTGTG \\
\hline
\end{tabular}

PIK3R1, phosphoinositide-3-kinase regulatory subunit 1; THBS1, thrombospondin-1; SPTAN1, nonerythrocytic $\alpha$-spectrin 1; SPP1, secreted phosphoprotein 1 .

(SPP1). The hazard ratio (HR), 95\% confidence interval (CI) and log-rank P-value were calculated and presented on the plot.

Patients and samples. A total of eight paired NSCLC and adjacent non-tumor lung tissue samples (within $5 \mathrm{~cm}$ of the tumor), including five squamous cancer and three adenocarcinoma samples, were obtained from patients who underwent surgery at The Second Affiliated Hospital of Xian Jiaotong University (Xian, China) between December 2017 and March 2018. Among these patients, six were male and two were female, and the median age was 59 years (range, 50-70 years). The present study was approved by the Ethics Committee of the Second Affiliated Hospital of Xian Jiaotong University (Xian, China). Written informed consent was obtained from all participants.

Reverse transcription-quantitative polymerase chain reaction (RT-qPCR) validation. Total RNA was extracted from tissues using Fast1000 (Shaanxi Pioneer Biotechnology Co., Ltd.), according to the manufacturer's protocol. A total $500 \mathrm{ng}$ total RNA was reverse transcribed in a final volume of $10 \mu \mathrm{l}$ using the PrimeScript ${ }^{\mathrm{TM}}$ RT reagent kit (Takara Biotechnology Co., Ltd., Dalian, China). The incubation conditions were as follows: $37^{\circ} \mathrm{C}$ for $15 \mathrm{~min}$ and $85^{\circ} \mathrm{C}$ for $5 \mathrm{sec}$, according to the manufacturer's protocols. TB Green ${ }^{\circledR}$ Premix Ex Taq ${ }^{\mathrm{TM}}$ II (Takara Biotechnology Co., Ltd.) was used for detecting the gene amplification and QPCR was performed on the CFX96 Touch ${ }^{\mathrm{TM}}$ Real-Time PCR Detection System (Bio-Rad Laboratories, Inc., Hercules, CA, USA). The thermocycling parameters were as follows: $95^{\circ} \mathrm{C}$ for $30 \mathrm{sec}$, followed by 40 cycles at $95^{\circ} \mathrm{C}$ for $5 \mathrm{sec}$ and $60^{\circ} \mathrm{C}$ for $30 \mathrm{sec}$. The primers for RT-qPCR are presented in Table I. The FC was calculated using the $2^{-\Delta \Delta C q}$ method and normalized to GAPDH expression (10). All experiments were performed in triplicate.

Statistical analysis. All data were analyzed using GraphPad Prism 8.0 (GraphPad Software, Inc., San Diego, CA, USA). 
Table II. A total of 230 DEGs were identified from microarray data, including 111 upregulated genes and 119 downregulated genes, in the non-small cell lung cancer high-metastatic cell line compared with the low-metastatic cell line.

Regulation

Upregulated

Downregulated

The results are presented as mean \pm standard deviation. Differences were analyzed by a paired Student's t-test. Association between gene expression and clinicopathological features was analyzed by $\chi^{2}$ test using SPSS 22.0 (IBM Corp., Armonk, NY, USA). $\mathrm{P}<0.05$ was considered to indicate a statistically significant difference.

\section{Results}

Identification of DEGs in NSCLC. Using microarray data, our previous study (6) identified 252 metastasis-associated mRNAs between the large-cell lung cancer high-metastatic 95D cell line and the low-metastatic 95C cell line, with FC $>2$ and $\mathrm{P}<0.05$. According to the NCBI database, it was identified that these mRNAs correspond to 230 genes, including 111 upregulated genes and 119 downregulated genes (Table II).

GO functional and KEGG pathway analysis. GO is a community-based bioinformatics resource that supplies information about gene product function using ontologies to represent biological knowledge (11). GO is often used to describe the biological roles of individual genomic products. GO consists of three aspects: Biological process (BP), cellular component (CC) and molecular function (MF) terms. BP terms refer to pathways and larger processes made up of the activities of multiple gene products. $\mathrm{CC}$ terms explain where gene products are active and MF terms indicate the molecular activities of

DEG, differentially expressed gene.

\section{DEG}

gene products. In order to identify the functional changes in the process of NSCLC metastasis, GO analysis was performed to analyze the functions of the DEGs. The results indicated that upregulated DEGs were most enriched in the following BP terms: 'Regulation of endothelial cell migration', 'negative regulation of neuron projection development' and 'endothelial cell migration'. The upregulated DEGs were enriched in the following CC terms: 'Plasma membrane', 'cell periphery' and 'membrane'. Furthermore, for MF terms, the upregulated DEGS were enriched in 'clathrin binding', 'phospholipase inhibitor activity' and 'lipase inhibitor activity'. Downregulated DEGs were enriched in the following BP terms: 'Response to protein stimulus', 'transforming growth factor beta receptor signaling pathway' and 'transmembrane receptor protein serine/threonine kinase signaling pathway'. The downregulated DEGs were enriched in the following CC terms: 'Cytoplasmic vesicle', 'extracellular matrix' and 'cell surface'. Finally, for MF terms, the downregulated DEGs were enriched in 'voltage-gated anion channel activity', 'calcium ion binding' and 'misfolded protein binding' (Fig. 1A-C). KEGG pathway enrichment analysis was also used to evaluate the possible pathways that the DEGs may be involved in. As presented in Fig. 1D, the upregulated DEGs were enriched in 'PI3K-Akt signaling pathway', 'cell adhesion molecules (CAMs)' and 'Jak-STAT signaling pathway', while the downregulated DEGs were enriched in 'leukocyte transendothelial migration', 'tight junction' and 'antigen processing and presentation'. 
A

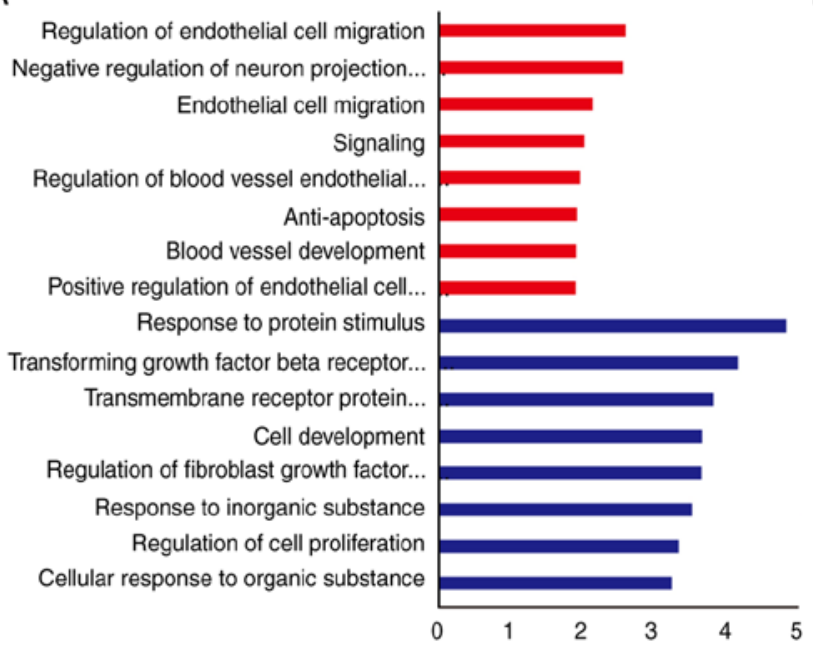

C

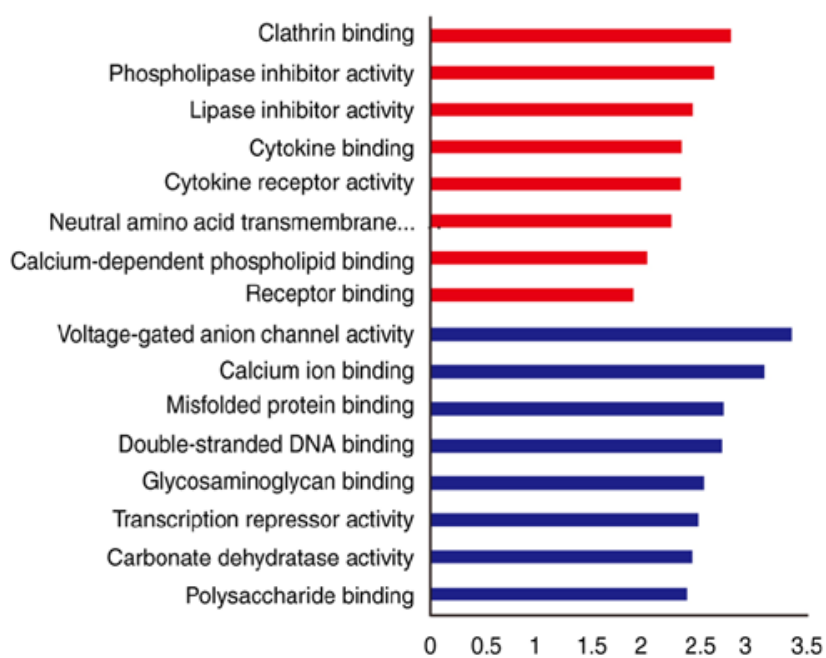

B

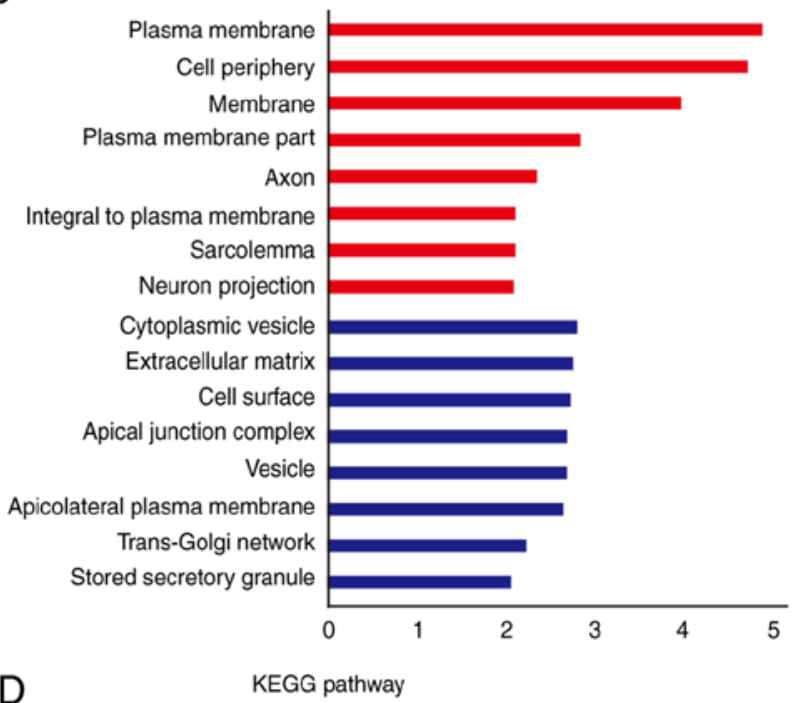

$\mathrm{D}$

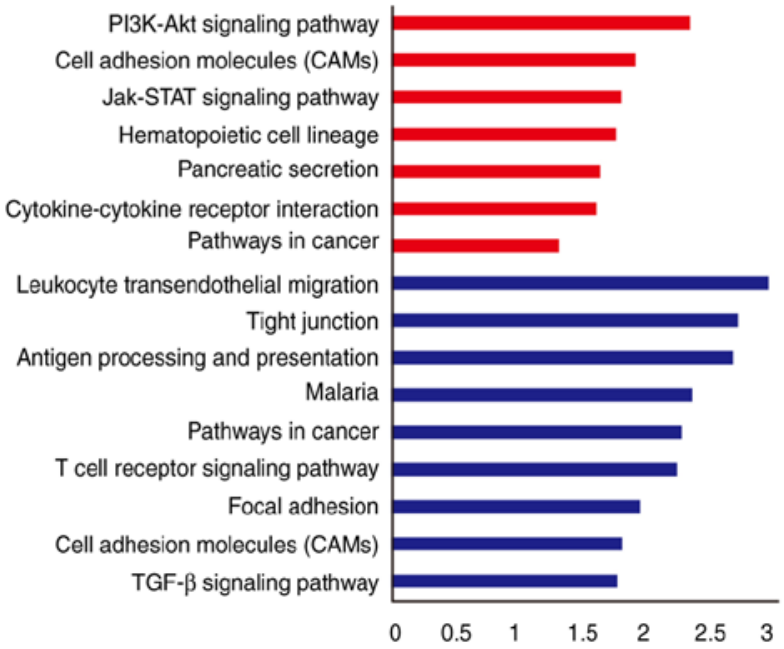

Figure 1. Gene Ontology and KEGG pathway analysis. (A) GO analysis of DEGs in biological process. (B) GO analysis of DEGs in cellular component. (C) GO analysis of DEGs in molecular function. (D) KEGG pathway analysis of DEGs. Red bars indicate upregulated genes and blue bars indicate downregulated genes. GO, Gene Ontology; KEGG, Kyoto Encyclopedia of Genes and Genomes; DEGs, differentially expressed genes.

PPInetwork and hub genes. The STRING database collects and integrates knowledge of the functional interactions between expressed proteins, while also predicting protein-protein association data for a large number of organisms (12). In the present study, STRING online database was used to illustrate the potential associations between the DEGs. Data were downloaded from STRING and mapped into Cytoscape software. A total of 230 DEGs were filtered into the PPI network complex, which included 101 nodes and 196 edges (Fig. 2A). The Cytoscape plug-in cytoHubba was applied to screen out the hub genes. The results indicated that among the 101 nodes, 17 central node genes (Table III) were identified with the criterion: Filtering degree $>7$ criteria, as each node had $>7$ connections. These 17 central node genes were termed the hub genes. The ten most significant node genes were phosphoinositide-3-kinase regulatory subunit 1 (PIK3R1), FOS, FYN, thrombospondin-1 (THBS1), nuclear receptor subfamily 3 group $\mathrm{C}$ member 1 , nonerythrocytic $\alpha$-spectrin 1 (SPTAN1), apolipoprotein $\mathrm{E}$, heat shock protein 1A, heat shock protein $90 \mathrm{kDa} \alpha$, class B member 1 and Annexin A1. Furthermore, two significant modules were selected from the
PPI network using MCODE. Module 1 consisted of seven nodes, including KISS1R, PIK3R1, PTGER1, TAC1, ANXA1, NTSR2, CHRM, and 21 edges (Fig. 2B). Module 2 consisted of five nodes, including ASB5, ZBTB16, ASB9, STUB1 and FBXL1 (Fig. 2C). The results of Module 1 and 2 GO term and KEGG pathway enrichment analysis are presented Tables SI and SII. In order to further analyze the hub genes, GO and KEGG pathway analysis was performed using the DAVID database. The results identified that the 17 hub genes predominantly participated in the 'response to drug' in $\mathrm{BP}$, 'cell surface' in CC, 'glycoprotein binding' in MF. KEGG analysis revealed that hub genes were enriched in 'PI3K-Akt signaling pathway', 'focal adhesion' and 'estrogen signaling pathway'.

Expression levels of hub genes in patients with lung cancer. GEPIA is a web-based tool that delivers fast and customizable functionalities based on TCGA and GTEx data (8). GEPIA provides key interactive and customizable functions, including differential expression analysis (8). To verify the reliability of the identified DEGs, the genes expression levels 


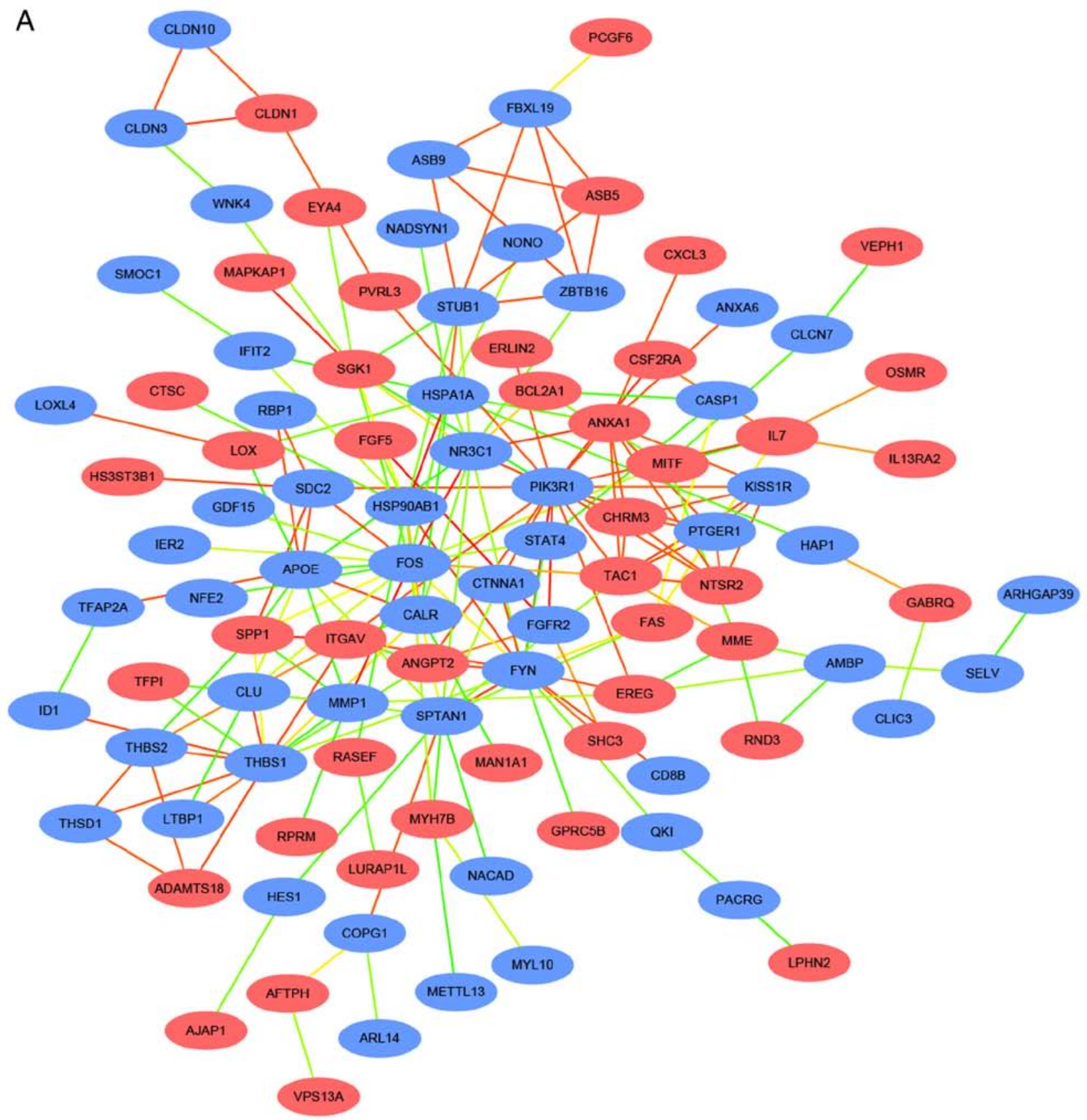

B

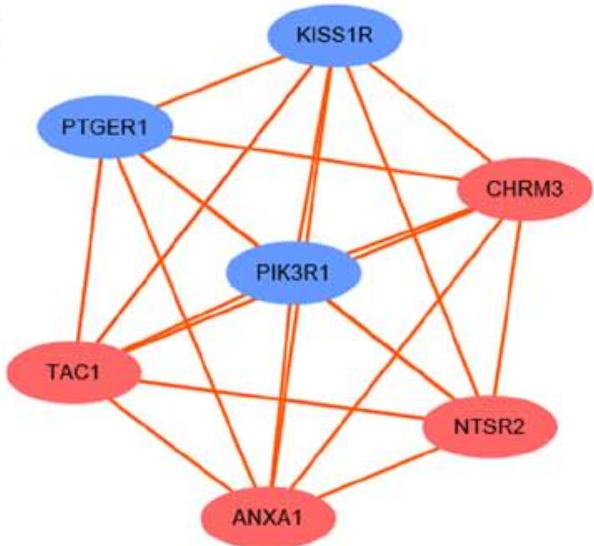

C

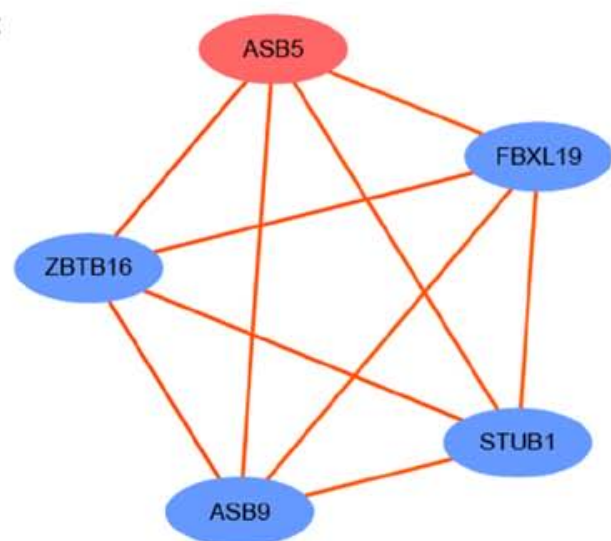

Figure 2. Protein-protein interaction network analysis. (A) A protein-protein interaction network for the DEGs. Red nodes represent upregulated DEGS and blue nodes represent downregulated DEGs. Red edges indicate a high combined score, followed by the yellow edges, and the green edges indicate the lowest combined score. Combined score indicates the strength of the correlation between the two genes. (B) Module 1 and (C) Module 2. DEGs, differentially expressed genes. 
Table III. Top 17 hub genes with the highest degrees of connectivity.

\begin{tabular}{lccr}
\hline Gene & $\begin{array}{c}\text { Degree of } \\
\text { connectivity }\end{array}$ & Fold-change & P-value \\
\hline PIK3R1 & 19 & -2.033 & 0.029 \\
FOS & 17 & -6.082 & 0.036 \\
FYN & 16 & -2.763 & 0.042 \\
THBS1 & 13 & -2.437 & 0.031 \\
NR3C1 & 12 & -2.709 & 0.005 \\
SPTAN1 & 12 & -2.201 & 0.011 \\
APOE & 11 & -9.232 & 0.001 \\
HSPA1A & 11 & -2.365 & 0.044 \\
HSP90AB1 & 10 & -2.382 & 0.002 \\
ANXA1 & 10 & 2.181 & 0.023 \\
TAC1 & 9 & 7.015 & 0.005 \\
SGK1 & 8 & 2.203 & 0.023 \\
STUB1 & 8 & -2.152 & 0.034 \\
SPP1 & 7 & -2.389 & 0.003 \\
MMP1 & 7 & -3.084 & 0.003 \\
ITGAV & 7 & 2.145 & $<0.001$ \\
CALR & 7 & -2.597 & 0.004 \\
\hline
\end{tabular}

were compared between patients with lung cancer and healthy individuals using GEPIA. As presented in Fig. 3, the expression levels of six genes, including PIK3R1, FOS, FYN, THBS1 and SPTAN1 were significantly lower in patients with lung adenocarcinoma (LUAD) or lung squamous cell carcinoma (LUSC) compared with healthy individuals, whereas secreted phosphoprotein 1 (SPP1) and matrix metalloproteinase-1 were significantly overexpressed in LUAD and LUSC.

Associations between hub gene expression levels and survival. The prognostic information of the 17 hub genes was evaluated using KM plotter. It was identified that the expression levels of PIK3R1 (HR, 0.58; 95\% CI, 0.51-0.66; $\left.\mathrm{P}<1 \times 10^{-16}\right)$, FYN (HR, 0.71; 95\% CI, 0.62-0.80; $\mathrm{P}=5.8 \times 10^{-8}$ ), SPTAN1 (HR, 0.81; 95\% CI, 0.71-0.92; $\mathrm{P}=0.0012$ ) and THBS1 (HR, 0.88; 95\% CI, 0.78-1.00; $\mathrm{P}=0.048$ ] were associated with increased OS time for patients with lung cancer. By contrast, SPP1 (HR, 1.32; 95\% 1.16-1.49; $\mathrm{P}=1.9 \times 10^{-5}$ ) was associated with poor OS time (Fig. 4).

Validation of DEGs. To verify whether the DEGs screened were reliable, RT-qPCR was used to detect the expression levels of these hub genes in eight pairs of lung cancer and adjacent tissue samples. The results revealed that the expression levels of PIK3R1, FYN, THBS1 and SPTAN1 were significantly lower in lung cancer tissues compared with adjacent normal lung tissues ( $\mathrm{P}<0.01$; Fig. 5). By contrast, SPP1 was expressed at a slightly higher level in lung cancer tissues $(\mathrm{P}=0.3743)$. This result was inconsistent with the microarray results; more clinical tissues are needed to validate the data in further studies. Subgroup analysis of the association between gene expression and clinicopathological features indicated that the expression of PIK3R1, FYN, THBS1 and SPTAN1 was lower in LUSC and LUAD, separately, compared with normal tissues. However, no association was observed between these genes and cancer stage, patient sex or age.

\section{Discussion}

Lung cancer is the most common malignancy worldwide, and the prognosis of lung cancer patients is very poor and 5 -year survival rate is $<15 \%$ (13). This is due to failure in early diagnosis and the occurrence of metastasis (14). Therefore, the detection of sensitive and specific biomarkers for lung cancer is required. To the best of our knowledge, the mechanism of lung cancer metastasis and invasion has not been fully reported. To investigate this underlying mechanism in our previous study (6), the gene microarray expression profiles were examined in the large-cell lung cancer high-metastatic 95D cell line and the low-metastatic 95C cell line. A total of 252 differentially expressed mRNAs, including 120 upregulated mRNAs and 132 downregulated mRNAs, were identified. These DEGs were subsequently analyzed by bioinformatics methods in the present study. GO functional analysis indicated that the majority of upregulated genes were involved in signaling and cell migration. A large number of these DEGs were located at the plasma membrane or cell periphery. Downregulated genes were mainly involved in 'response to protein stimulus', 'cytoplasmic vesicle' and 'voltage-gated anion channel activity'. The KEGG pathway analysis indicated that upregulated DEGs were associated with 'PI3K-Akt signaling pathway' and 'cell adhesion molecules (CAMs)', while the downregulated DEGs were predominantly enriched in 'leukocyte transendothelial migration' and 'tight junction'.

The STRING online database and Cytoscape software were used to construct a PPI network. According to the degree of connectivity, 17 hub genes were filtered out. Among them, five hub genes were closely associated with the OS of patients with lung cancer, according to KM plotter. In addition, these genes, including PIK3R1, FYN, THBS1, SPTAN1 and SPP1, were significantly differentially expressed between healthy individuals and patients with cancer based on the GEPIA database. The expression levels of five genes were verified using eight paired lung cancer tissue and normal lung tissue samples by RT-qPCR.

The PI3K/AKT pathway is one of the most understood cancer-associated pathways. Class IA PI3Ks are widely studied heterodimers that include a catalytic subunit and a regulatory subunit. The catalytic subunit p110a is encoded by phosphatidylinositol-4,5-bisphosphate 3-kinase catalytic subunit $\alpha$, while the regulatory subunit $\mathrm{p} 85 \mathrm{a}$ is encoded by PIK3R1 (15). P85 $\alpha$ not only inhibits the kinase catalytic activity of p110 $\alpha$, but also binds to PTEN, preventing PTEN ubiquitination and increasing its stability (16). Mutations in PIK3R1 are implicated in cases of breast cancer (17).

FYN, a member of the Src family tyrosine kinases, is associated with $\mathrm{T}$ cell and neuronal signaling in development and normal cellular physiology (18). FYN is an oncogene, which encodes a protein that is highly expressed in various types of cancer (19-21). FYN is associated with epithelial-mesenchymal transition (22) and also participates in a number of cancer metastasis-associated pathways, including the TGF $\beta$ 

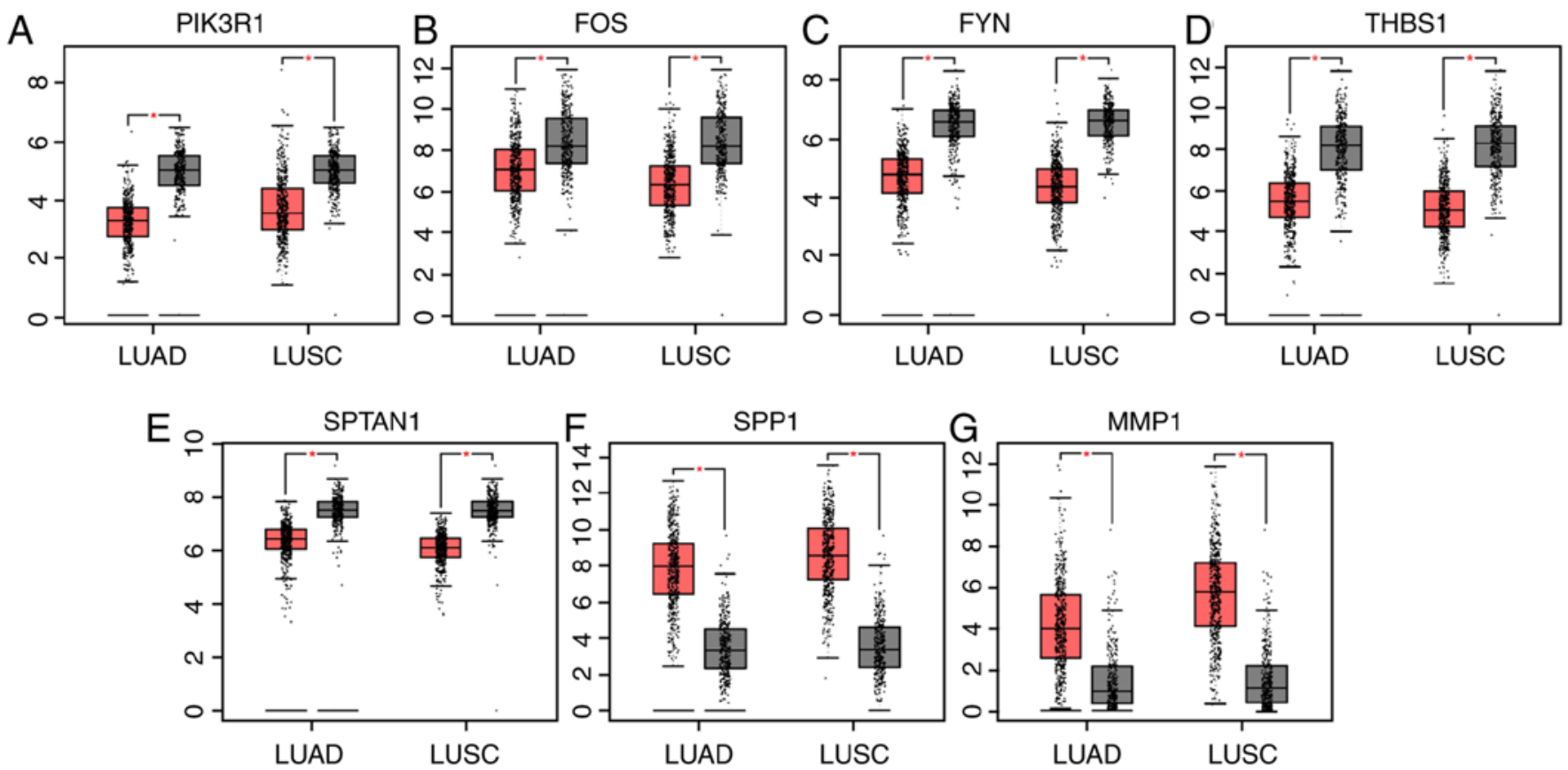

Figure 3. Expression levels of seven genes in patients with lung cancer and healthy individuals using data from Gene Expression Profiling Interactive Analysis. (A) PIK3R1, (B) FOS, (C) FYN, (D) THBS1, (E) SPTAN1, (F) SPP1 and (G) MMP1 expression levels in patients with LUAD and LUSC, and healthy individuals. Red, patients with LUAD or LUSC; grey, healthy individuals. " $\mathrm{P}<0.05$. LUAD, lung adenocarcinoma; LUSC, lung squamous cell carcinoma; PIK3R1, phosphoinositide-3-kinase regulatory subunit 1; THBS1, thrombospondin-1; SPTAN1, nonerythrocytic $\alpha$-spectrin 1; SPP1, secreted phosphoprotein 1; MMP1, matrix metalloproteinase 1 .
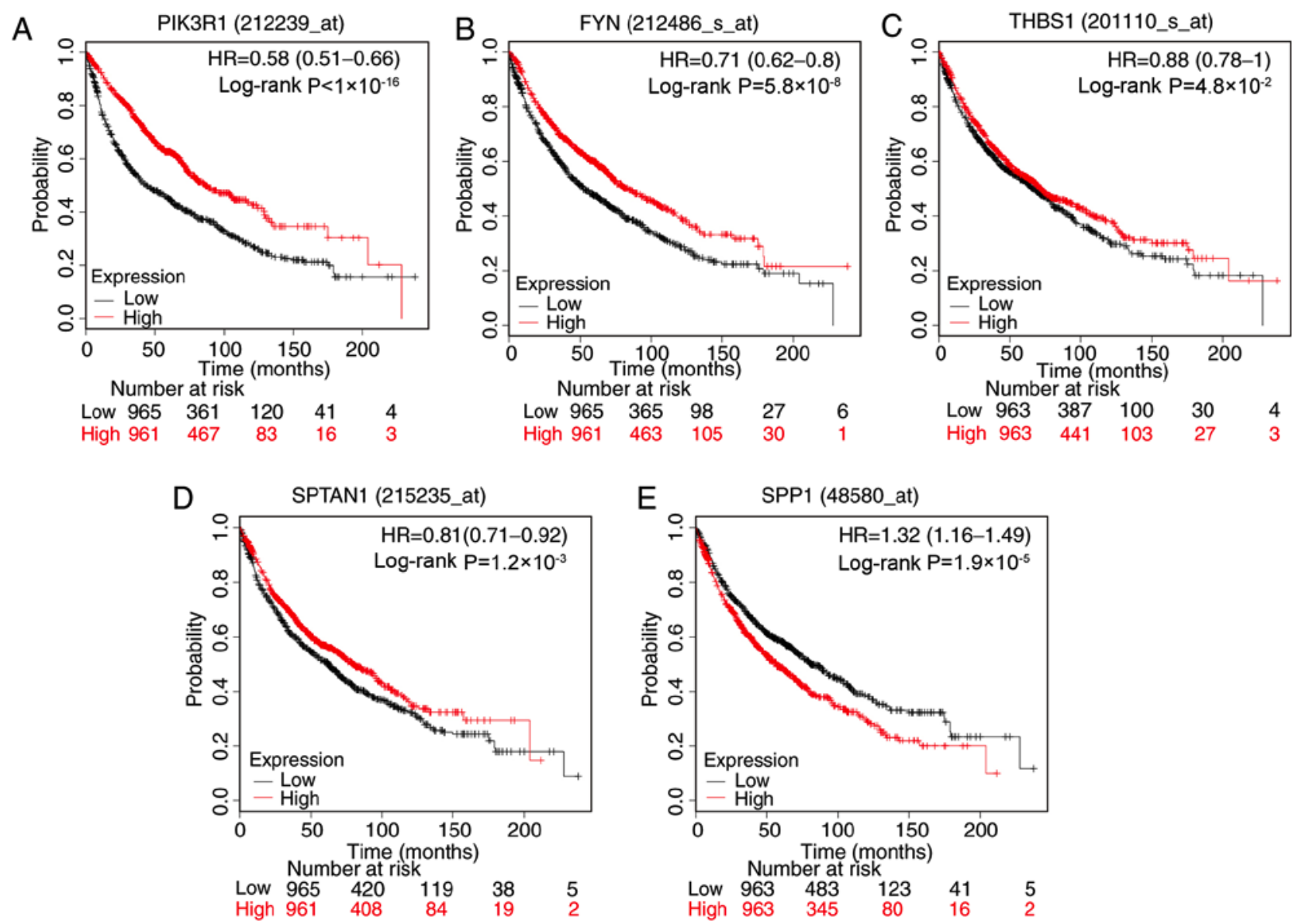

Figure 4. Prognostic value of five genes. Survival curves of patients with lung cancer according to the expression level of (A) PIK3R1, (B) FYN, (C) THBS1, (D) SPTAN1 and (E) SPP1. The valid Affymetrix IDs were as follows: 212239_at (PIK3R1), 212486_s_at (FYN), 201110_s_at (THBS1), 215235_at (SPTAN1), 48580_at (SPP1). HR, hazard ratio; PIK3R1, phosphoinositide-3-kinase regulatory subunit 1; THBS1, thrombospondin-1; SPTAN1, nonerythrocytic $\alpha$-spectrin 1; SPP1, secreted phosphoprotein 1 . 

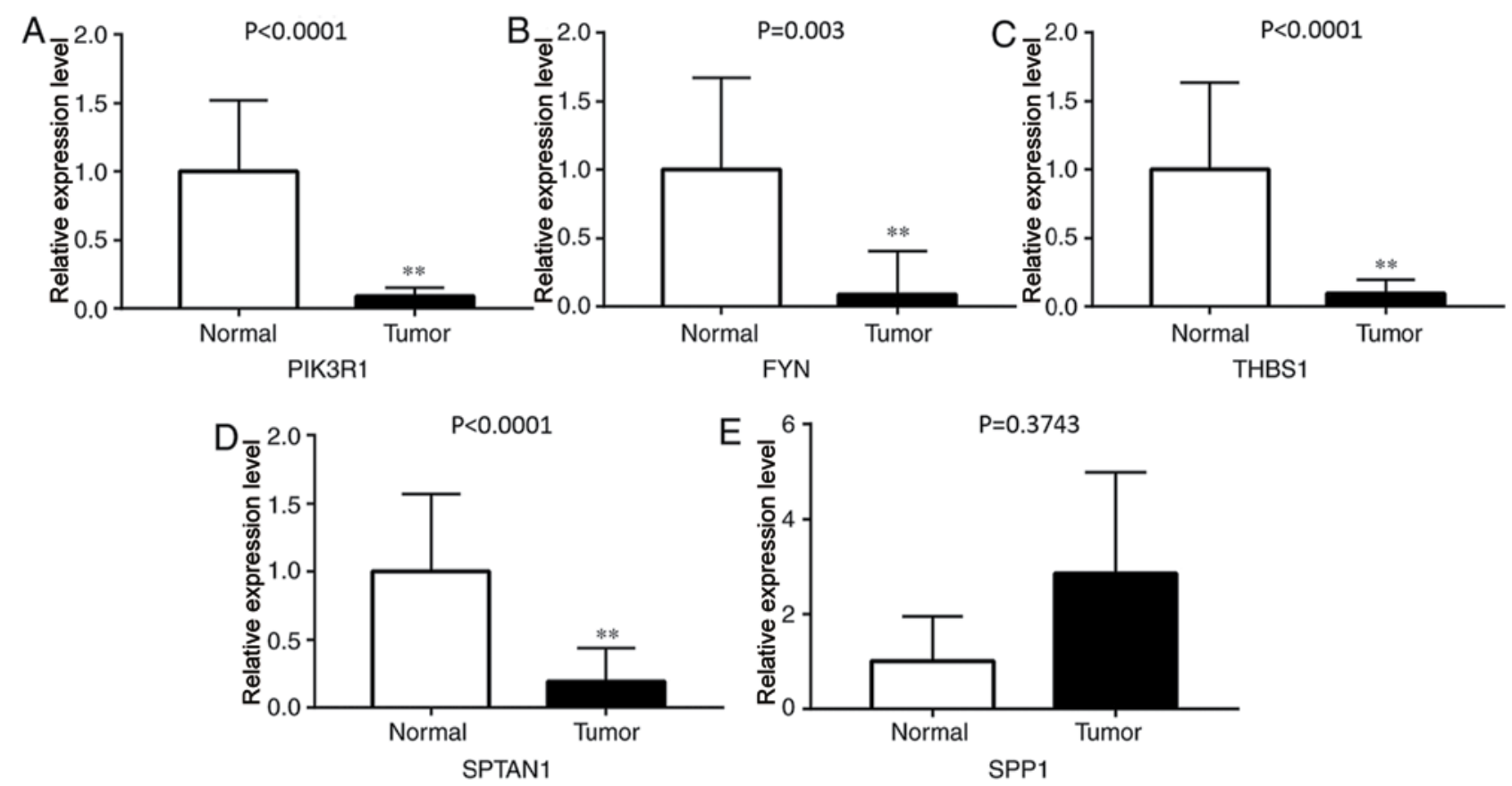

Figure 5. Expression of PIK3R1, FYN, THSB1, SPTAN1 and SPP1 in lung cancer tissues and normal lung tissues. (A-D) The expression of (A) PIK3R1, (B) FYN, (C) THBS1 and (D) SPTAN1 was significantly lower in lung cancer tissues compared with that in normal lung tissues. (E) SPP1 expression was higher in lung cancer tissues compared with that in normal lung tissues. ${ }^{* *} \mathrm{P}<0.01$ vs. normal lung tissues. PIK3R1, phosphoinositide-3-kinase regulatory subunit 1; THBS1, thrombospondin-1; SPTAN1, nonerythrocytic $\alpha$-spectrin 1; SPP1, secreted phosphoprotein 1.

pathway (23). In the present study, FYN was expressed at a low level in high metastatic cancer cells, while the database analysis indicated that high FYN expression was associated with an improved OS time. These results are contradictory to previous studies (19-21); further studies need to be done in the future.

THBS1 was first identified in 1971 as a member of the extracellular matrix protein family (24). As an angiogenesis inhibitor, THBS1 regulates diverse processes, including adhesion, invasion, migration, proliferation and apoptosis in numerous types of cancer, such as hepatocellular carcinoma, lung and breast cancer $(25,26)$. However, the precise role of THBS1 in tumor invasion and migration remains controversial. A number of studies have suggested that THBS1 exhibits both stimulatory and inhibitory roles in different tumor types. On the one hand, THBS1 has been reported to stimulate the expression of metalloproteinases partly via the integrin signaling pathway, and enhance the invasion and migration in oral squamous cell carcinoma cells (27). On the other hand, THBS1 has been demonstrated to inhibit the migration of clear cell renal carcinoma cells in response to different stimuli (28). The present data indicated that THBS1 may serve as a suppressor gene in lung cancer.

Spectrin, a cytoskeletal protein, serves an important role in maintaining the stability, structure and shape of the cell membrane (29). It has two $\alpha$ subunits and five $\beta$ subunits, including $\alpha \mathrm{I}, \alpha \mathrm{II}, \beta \mathrm{I}, \beta \mathrm{II}, \beta \mathrm{III}, \beta \mathrm{IV}$ and $\beta \mathrm{V}$. SPTAN1 encodes a number of $\alpha \mathrm{II}$-spectrin isoforms that are expressed in all nonerythroid cells (30). Recent studies have reported that a dysregulation of SPTAN1 effects cellular behavior and promotes tumor progression $(31,32)$. A study reported that SPTAN1 had recurrent mutations in 27 lung adenocarcinomas of individuals that had never smoked, and these mutations were highly associated with pathway dysregulation and patient survival (33).

SPP1, also termed osteopontin (OPN), is a 41-75 $\mathrm{kDa}$ extracellular matrix phosphoprotein. SPP1 is a member of the small integrin binding ligand $\mathrm{N}$-linked glycoproteins family and it is expressed in multiple tissues, particularly in bones (34). SPP1 can regulate tumor invasion and metastasis by binding and activating matrix metalloproteinases (35). In addition, SPP1 has been reported to be abnormally expressed in a variety of tumor types. For instance, SPP1 is highly expressed in liver cancer and may be a prognostic and diagnostic marker of HCC (36). The expression of SPP1 is higher in epithelial ovarian cancer tissues compared with that in normal ovarian tissues, and silencing SPP1 decreased the cell proliferation, migration, and invasion; these effects may be dependent on the integrin $\beta 1 / F A K / A K T$ signaling pathway (37). In lung cancer, a high expression of SPP1 is associated with tumor stage, lymph node invasion and tumor growth (38). A recent study revealed that OPN-a, a splicing variant, increased A549 cell adherent abilities to bone tissues by interacting with the cell surface receptor $\alpha v \beta 3$ integrin. Therefore, OPN-a may represent a bone metastatic factor and a potential therapeutic target in human lung cancer (39). Despite the fact that there was no significant difference in the expression of SPP1 between lung cancer tissues and normal lung tissues in the present study, SPP1 may still serve as a good diagnostic marker and future studies are required to investigate this further.

In conclusion, the present study identified DEGs that may serve a role in the invasion and metastasis of NSCLC using bioinformatics methods. A total of 230 DEGs and 17 hub genes were selected, and PIK3R1, FYN, THBS1, SPTAN1 and SPP1 were identified to be potential core genes of NSCLC. In order 
to obtain more accurate association results, a larger number of clinical specimens is required to perform further verification experiments. In summary, the current study may provide novel insights that assist with the development of individualized treatments for NSCLC.

\section{Acknowledgements}

Not applicable.

\section{Funding}

The present study was supported by National Natural Science Foundation of China (grant no. 81672300) and Shaanxi Science and Technology Development Research Program of China (grant no. 2016YFJH2-11).

\section{Availability of data and materials}

The datasets used during the present study are available from the corresponding author on reasonable request.

\section{Authors' contributions}

RS contributed to the conception of the study and wrote the manuscript. XM, WW, BL performed the bioinformatics analysis. JY and LZ performed reverse transcription-quantitative PCR. XL, YC and BY analyzed the data. SY performed the bioinformatics analysis and critically revised and checked the manuscript. All authors read and approved the final manuscript.

\section{Ethics approval and consent to participate}

The present study was approved by the Ethics Committee of the Second Affiliated Hospital of Xi'an Jiaotong University (Xi'an, China). Written informed consent was obtained from all participants.

\section{Patient consent for publication}

Not applicable.

\section{Competing interests}

The authors declare that there are no competing interests.

\section{References}

1. Siegel RL, Miller KD and Jemal A: Cancer statistics, 2019. CA Cancer J Clin 69: 7-34, 2019.

2. Goldstraw P, Ball D, Jett JR, Le Chevalier T, Lim E, Nicholson AG and Shepherd FA: Non-small-cell lung cancer. Lancet 378: 1727-1740, 2011

3. Brody H: Lung cancer. Nature 513 (Suppl): S1, 2014.

4. Malone JH and Oliver B: Microarrays, deep sequencing and the true measure of the transcriptome. BMC Biol 9: 34, 2011.

5. Allison DB, Cui X, Page GP and Sabripour M: Microarray data analysis: From disarray to consolidation and consensus. Nat Rev Genet 7: 55-65, 2006.

6. Zequn N, Xuemei Z, Wei L, Zongjuan M, Yujie Z, Yanli H, Yuping Z, Xia M, Wei W, Wenjing D, et al: The role and potential mechanisms of LncRNA-TATDN1 on metastasis and invasion of non-small cell lung cancer. Oncotarget 7: 18219-18228, 2016
7. Huang da W, Sherman BT and Lempicki RA: Systematic and integrative analysis of large gene lists using DAVID bioinformatics resources. Nat Protoc 4: 44-57, 2009.

8. Tang Z, Li C, Kang B, Gao G, Li C and Zhang Z: GEPIA: A web server for cancer and normal gene expression profiling and interactive analyses. Nucleic Acids Res 45: W98-W102, 2017.

9. Győrffy B, Surowiak P, Budczies J and Lanczky A: Online survival analysis software to assess the prognostic value of biomarkers using transcriptomic data in non-small-cell lung cancer. PLoS One 8: e82241, 2013.

10. Livak KJ and Schmittgen TD: Analysis of relative gene expression data using real-time quantitative PCR and the 2(-Delta Delta C(T)) method. Methods 25: 402-408, 2001.

11. Gene Ontology Consortium: Gene ontology consortium: Going forward. Nucleic Acids Res 43: D1049-D1056, 2015.

12. Szklarczyk D, Morris JH, Cook H, Kuhn M, Wyder S, Simonovic M, Santos A, Doncheva NT, Roth A, Bork P, et al: The STRING database in 2017: Quality-controlled protein-protein association networks, made broadly accessible. Nucleic Acids Res 45: D362-D368, 2017.

13. Jamal-Hanjani M, Wilson GA, McGranahan N, Birkbak NJ, Watkins TBK, Veeriah S, Shafi S, Johnson DH, Mitter R, Rosenthal R, et al: Tracking the evolution of non-small-cell lung cancer. N Engl J Med 376: 2109-2121, 2017.

14. Hamilton G and Rath B: Mesenchymal-epithelial transition and circulating tumor cells in small cell lung cancer. Adv Exp Med Biol 994: 229-245, 2017.

15. Kriplani N, Hermida MA, Brown ER and Leslie NR: Class I PI 3-kinases: Function and evolution. Adv Biol Regul 59: 53-64, 2015.

16. Chagpar RB, Links PH, Pastor MC, Furber LA, Hawrysh AD, Chamberlain MD and Anderson DH: Direct positive regulation of PTEN by the p85 subunit of phosphatidylinositol 3-kinase. Proc Natl Acad Sci USA 107: 5471-5476, 2010.

17. Chen L, Yang L, Yao L, Kuang XY, Zuo WJ, Li S, Qiao F, Liu YR, Cao ZG, Zhou SL, et al: Characterization of PIK3CA and PIK3R1 somatic mutations in Chinese breast cancer patients. Nat Commun 9: 1357, 2018.

18. Saito YD, Jensen AR, Salgia R and Posadas EM: Fyn: A novel molecular target in cancer. Cancer 116: 1629-1637, 2010.

19. Li X, Yang Y, Hu Y, Dang D, Regezi J, Schmidt BL, Atakilit A, Chen B, Ellis D and Ramos DM: Alphavbeta6-Fyn signaling promotes oral cancer progression. J Biol Chem 278: 41646-41653, 2003.

20. Posadas EM, Al-Ahmadie H, Robinson VL, Jagadeeswaran R, Otto K, Kasza KE, Tretiakov M, Siddiqui J, Pienta KJ, Stadler WM, et al: FYN is overexpressed in human prostate cancer. BJU Int 103: 171-177, 2009.

21. Zhang X, Li F and Zhu PL: Fyn-related kinase expression predicts favorable prognosis in patients with cervical cancer and suppresses malignant progression by regulating migration and invasion. Biomed Pharmacother 84: 270-276, 2016.

22. Lewin B, Siu A, Baker C, Dang D, Schnitt R, Eisapooran P and Ramos DM: Expression of Fyn kinase modulates EMT in oral cancer cells. Anticancer Res 30: 2591-2596, 2010.

23. Kim AN, Jeon WK, Lim KH, Lee HY, Kim WJ and Kim BC: Fyn mediates transforming growth factor-beta1-induced down-regulation of E-cadherin in human A549 lung cancer cells. Biochem Biophys Res Commun 407: 181-184, 2011.

24. Li Y, Turpin CP and Wang S: Role of thrombospondin 1 in liver diseases. Hepatol Res 47: 186-193, 2017.

25. Huang T, Sun L, Yuan X and Qiu H: Thrombospondin-1 is a multifaceted player in tumor progression. Oncotarget 8: 84546-84558, 2017.

26. Jeanne A, Schneider C, Martiny L and Dedieu S: Original insights on thrombospondin-1-related antireceptor strategies in cancer. Front Pharmacol 6: 252, 2015.

27. Pal SK, Nguyen CT, Morita KI, Miki Y, Kayamori K, Yamaguchi A and Sakamoto K: THBS1 is induced by TGFB1 in the cancer stroma and promotes invasion of oral squamous cell carcinoma. J Oral Pathol Med 45: 730-739, 2016.

28. Bienes-Martinez R, Ordonez A, Feijoo-Cuaresma M, Corral-Escariz M, Mateo G, Stenina O, Jiménez B and Calzada MJ: Autocrine stimulation of clear-cell renal carcinoma cell migration in hypoxia via HIF-independent suppression of thrombospondin-1. Sci Rep 2: 788, 2012.

29. Hartwig JH: Actin-binding proteins 1: Spectrin superfamily. Protein Profile 1: 706-778, 1994.

30. Cianci CD, Zhang Z, Pradhan D and Morrow JS: Brain and muscle express a unique alternative transcript of alphaII spectrin. Biochemistry 38: 15721-15730, 1999. 
31. Ackermann A, Schrecker C, Bon D, Friedrichs N, Bankov K, Wild P, Plotz G, Zeuzem S, Herrmann E, Hansmann ML and Brieger A: Downregulation of SPTAN1 is related to MLH1 deficiency and metastasis in colorectal cancer. PLoS One 14: e0213411, 2019.

32. Sheng G, Zhang J, Zeng Z, Pan J, Wang Q, Wen L, Xu Y, Wu D and Chen S: Identification of a novel CSF3R-SPTAN1 fusion gene in an atypical chronic myeloid leukemia patient with $\mathrm{t}(1 ; 9)$ (p34;q34) by RNA-Seq. Cancer Genet 216-217: 16-19, 2017.

33. Sun Z, Wang L, Eckloff BW, Deng B, Wang Y, Wampfler JA, Jang J, Wieben ED, Jen J, You M and Yang P: Conserved recurrent gene mutations correlate with pathway deregulation and clinical outcomes of lung adenocarcinoma in never-smokers. BMC Med Genomics 7: 32, 2014.

34. Wei R, Wong JPC and Kwok HF: Osteopontin-a promising biomarker for cancer therapy. J Cancer 8: 2173-2183, 2017.

35. Fedarko NS, Jain A, Karadag A and Fisher LW: Three small integrin binding ligand N-linked glycoproteins (SIBLINGs) bind and activate specific matrix metalloproteinases. FASEB J 18 : 734-736, 2004

36. Cabiati M, Gaggini M, Cesare MM, Caselli C, De Simone P, Filipponi F, Basta G, Gastaldelli A and Del Ry S: Osteopontin in hepatocellular carcinoma: A possible biomarker for diagnosis and follow-up. Cytokine 99: 59-65, 2017.
37. Zeng B, Zhou M, Wu H and Xiong Z: SPP1 promotes ovarian cancer progression via Integrin beta1/FAK/AKT signaling pathway. Onco Targets Ther 11: 1333-1343, 2018

38. Hu Z, Lin D, Yuan J, Xiao T, Zhang H, Sun W, Han N, Ma Y, Di X, Gao M, et al: Overexpression of osteopontin is associated with more aggressive phenotypes in human non-small cell lung cancer. Clin Cancer Res 11: 4646-4652, 2005.

39. Hao C, Cui Y, Hu MU, Zhi X, Zhang L, Li W, Wu W, Cheng S and Jiang WG: OPN-a splicing variant expression in non-small cell lung cancer and its effects on the bone metastatic abilities of lung cancer cells in vitro. Anticancer Res 37: 2245-2254, 2017.

This work is licensed under a Creative Commons Attribution-NonCommercial-NoDerivatives 4.0 International (CC BY-NC-ND 4.0) License. 